\title{
Computational study on Hydroxybenzotriazoles as Reagents for Ester
}

\section{Hydrolysis}

\author{
V. Praveen Kumar, Biswajit Ganguly ${ }^{*}$ and Santanu Bhattacharya ${ }^{*}$
}

\section{Table of Contents:}

Scheme S1

Synthesis

TableS1

Table S2a

Table S2b

Table S3a

Table S3b

Table S4

Tabel S5

Cartesian coordinates of full-optimized geometries
$\mathrm{S} 2$

$\mathrm{S} 2-\mathrm{S} 3$

S4

S5

S6

S7

S8

S9

S10

S11-S21 


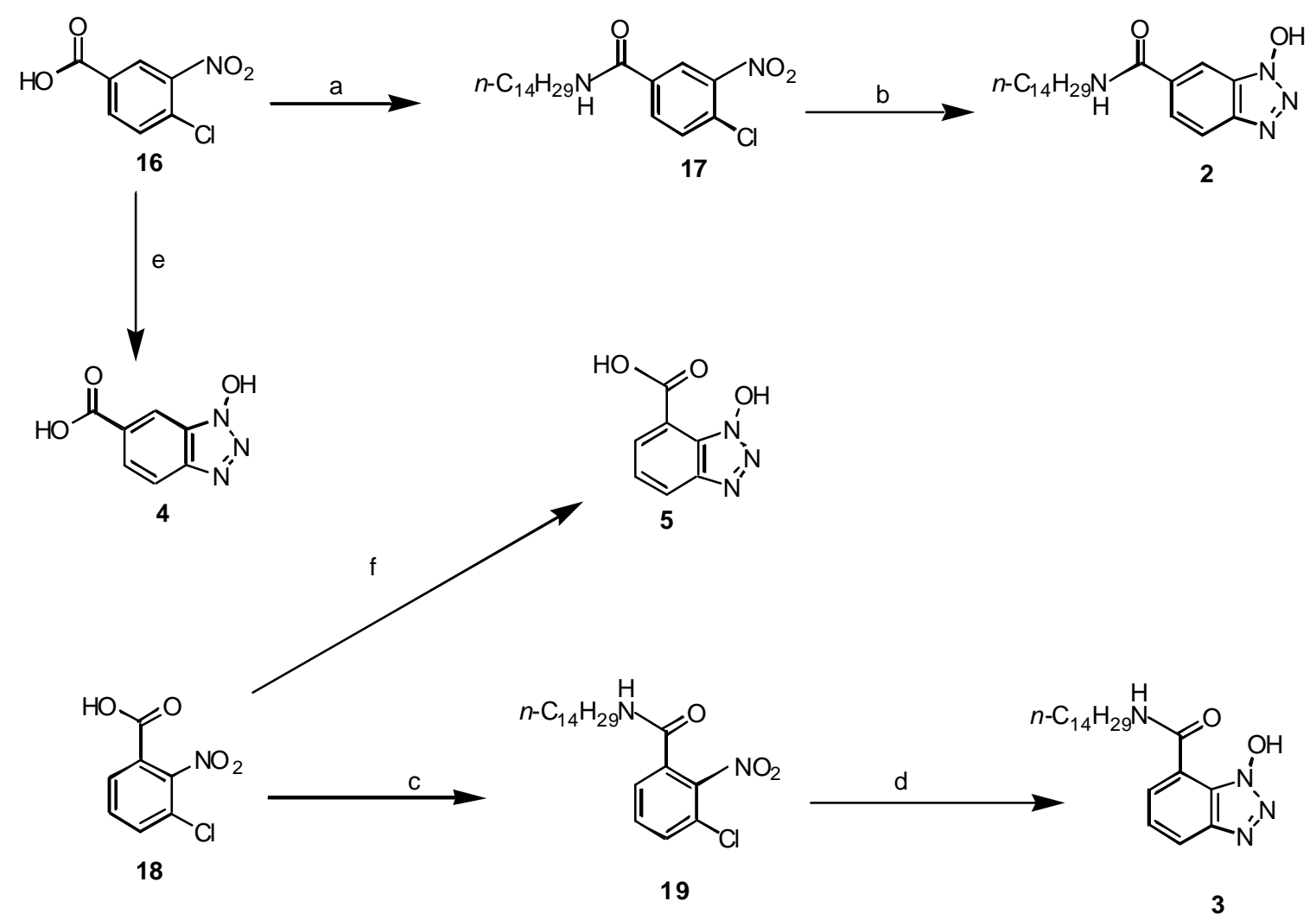

${ }^{a}$ Reagents and conditions: (a) (i) $\mathrm{SOCh}_{2}$, reflux, $3 \mathrm{~h}$; (ii) $n$-tetradecylamine (1.2 equiv.), $\mathrm{Et}_{3} \mathrm{~N}$, dry THF, $-10{ }^{\circ} \mathrm{C}$ (30 min), rt, 8 h, $82 \%$ (b) $\mathrm{N}_{2} \mathrm{H}_{4} \cdot \mathrm{H}_{2} \mathrm{O}$, dry EtOH, reflux, $20 \mathrm{~h}, 60 \%$; (c) (i) $\mathrm{SOCh}$, reflux, $3 \mathrm{~h}$; (ii) $n$-tetradecylamine (1.2 equiv.), $\mathrm{Et}_{3} \mathrm{~N}$, dry THF, $-10{ }^{\circ} \mathrm{C}\left(30 \mathrm{~min}\right.$ ), rt, $8 \mathrm{~h}, 80 \%$ (d) $\mathrm{N}_{2} \mathrm{H}_{4} \cdot \mathrm{H}_{2} \mathrm{O}$, dry EtOH, reflux, $48 \mathrm{~h}, 60 \%$; (e) $\mathrm{N}_{2} \mathrm{H}_{4} \cdot \mathrm{H}_{2} \mathrm{O}$, dry $\mathrm{EtOH}$, reflux, 15 h, 76\%. (f) $\mathrm{N}_{2} \mathrm{H}_{4} \cdot \mathrm{H}_{2} \mathrm{O}$, dry EtOH, reflux, $18 \mathrm{~h}$, $62 \%$.

\section{Synthesis.}

$N$-1-Tetradecyl-4-chloro-3-nitrobenzamide (17). 4-Chloro-3-nitrobenzoic acid, 16 (1 g, 5 mmol) was dissolved in $15 \mathrm{~mL}$ of distilled $\mathrm{SOCh}$ and the mixture was heated to reflux for $3 \mathrm{~h}$. Then the reaction mixture was allowed to cool, and the excess $\mathrm{SOCh}$ was removed in vacuum. This afforded light yellow crystals, and which showed characteristic peak at $v_{\max } 1782 \mathrm{~cm}^{-1}$ in IR. The yellowish solid was dissolved in freshly dried THF $(10 \mathrm{~mL})$ in the presence of $\mathrm{Et}_{3} \mathrm{~N}(0.83 \mathrm{~mL}, 6 \mathrm{mmol})$. To this $n$ tetradecylamine $(1.27 \mathrm{~mL}, 6 \mathrm{mmol})$ was added at $-10{ }^{\circ} \mathrm{C}$, and the reaction mixture was stirred for $30 \mathrm{~min}$ at $-10{ }^{0} \mathrm{C}$, and then gradually warmed to room temparature and stirring was continued for another $8 \mathrm{~h}$. THF was evaporated from the reaction mixture and then $25 \mathrm{~mL}$ of dilute $\mathrm{HCl}(\mathrm{pH} 5)$ was added. The mixture was extracted thoroughly with ethyl acetate $(3 \times 25 \mathrm{~mL})$. Organic layer was dried over anhyd. $\mathrm{Na}_{2} \mathrm{SO}_{4}$. Evaporation of the organic solvent gave a crude solid, which was purified by column chromatography over silica gel using EtOAc/hexane (15:85) to furnish $\mathbf{1 7}$ as a solid (1.61 g, $82 \%)$; mp 74-75 ${ }^{\circ} \mathrm{C}$; IR (nujol), $v_{\max } 3320,1642,1537,1338 \mathrm{~cm}^{-1} ;{ }^{1} \mathrm{H}-\mathrm{NMR}\left(\mathrm{CDCl}_{3}, 300 \mathrm{MHz}\right) \delta 8.18(\mathrm{~d}, 1 \mathrm{H})$, $7.96(\mathrm{dd}, 1 \mathrm{H}), 7.64(\mathrm{~d}, 1 \mathrm{H}), 6.1$ (br s, 1H), 3.47 (q, 2H), $1.61(\mathrm{~m}, 2 \mathrm{H}), 1.25(\mathrm{~s}, 22 \mathrm{H}), 0.88(\mathrm{t}, 3 \mathrm{H})$; EIMS; m/z $396\left(\mathrm{M}^{+}\right)$. 
$\boldsymbol{N}$-6-Tetradecyl-1-hydroxy-1H-benzo[d][1,2,3]triazolecarboxamide (2). A mixture of 17 (1.58 g, 4 mmol) and hydrazine hydrate $(1.94 \mathrm{~mL}, 40 \mathrm{mmol})$ in dry EtOH was heated under reflux for $20 \mathrm{~h}$ and then cooled to room temperature. From the reaction mixture, EtOH was evaporated under reduced pressure. This left a residue to which $\mathrm{MeOH}(20 \mathrm{~mL})$ was added and the mixture was acidified with conc. HCl. This induced precipitation of a yellowish white solid. This was filtered and solvent from the filtrate upon evaporation afforded additional amount of yellowish solid. The residues were combined and were taken up in $\mathrm{CHCl}_{3}$. The chloroform solution was washed repeatedly with water and phase separated. Then the organic layer was dried over anhyd. $\mathrm{Na}_{2} \mathrm{SO}_{4}$, the solvent was evaporated to afford a yellowish solid which was further purified by column chromatography over silica gel with using $\mathrm{MeOH} / \mathrm{EtOAc}(2 / 98)$ to give 2 as a yellowish solid $(0.89 \mathrm{~g}, 60 \%)$; mp $141-142{ }^{\circ} \mathrm{C}$; IR $(\mathrm{KBr}), v_{\max }$, 3422, 3336, 2919, 1634, 1544, $856 \mathrm{~cm}^{-1} ;{ }^{1} \mathrm{H}-\mathrm{NMR}\left(\mathrm{CDCl}_{3} / \mathrm{DMSO}_{6}, 300 \mathrm{MHz}\right) \delta 8.16(\mathrm{~d}, 1 \mathrm{H}), 7.89$ (dd, 2H), 4.7 (br s, 1H), 3.45 (q, 2H), $1.64(\mathrm{~m}, 2 \mathrm{H}), 1.25(\mathrm{~s}, 22 \mathrm{H}), 0.87(\mathrm{t}, 3 \mathrm{H})$; EI-MS; m/z $357\left(\mathrm{M}^{+}\right.$17); Anal. Calcd for $\mathrm{C}_{21} \mathrm{H}_{34} \mathrm{~N}_{4} \mathrm{O}_{2}$ : C, 67.34; H, 9.15; N, 14.95. Found: C, 67.24; H, 9.46; N, 14.54.

N-1-Tetradecyl-3-chloro-2-nitrobenzamide (19). Compound 19 was synthesized using a similar procedure adopted for the synthesis of 17. From $0.2 \mathrm{~g}(1 \mathrm{mmol})$ of 3-chloro-2-nitrobenzoic acid, 8, compound 9 was obtained as a light yellowish solid $(0.32 \mathrm{~g})$ in $82 \%$ yield. mp $62-64{ }^{\circ} \mathrm{C}$; IR $(\mathrm{KBr}), v_{\max }$ 3287, 1651, 1539, $1351 \mathrm{~cm}^{-1}$; ${ }^{1} \mathrm{H}-\mathrm{NMR}\left(\mathrm{CDCl}_{3}, 300 \mathrm{MHz}\right) \delta 7.58(\mathrm{~m}, 3 \mathrm{H}), 5.96(\mathrm{br}, 1 \mathrm{H}), 3.40(\mathrm{q}, 2 \mathrm{H})$, 1.25 (s, 24H), 0.88 (t, 3H); EI-MS; m/z $397\left(\mathrm{M}^{+}\right)$.

$\boldsymbol{N}$-7-Tetradecyl-1-hydroxy-1H-benzo[d][1,2,3]triazole-7-carboxamide $\quad(3)$. Compound 3 was synthesized using a similar procedure as described in the synthesis of 2. Starting with $0.29 \mathrm{~g}(0.75$ mmol) of 9, we obtained 3 as a solid $(0.16 \mathrm{~g}, 60 \%)$; mp 128-130 ${ }^{\circ} \mathrm{C}$; IR (KBr), $v_{\max } 3437,3068,2919$, 1643, 1573, $864 \mathrm{~cm}^{-1}$; ${ }^{1} \mathrm{H}-\mathrm{NMR}\left(\mathrm{CDCl}_{3} / \mathrm{DMSO}_{6}, 300 \mathrm{MHz}\right) \delta 8.07(\mathrm{~d}, 1 \mathrm{H}), 7.95(\mathrm{~d}, 1 \mathrm{H}), 7.31(\mathrm{t}$, 1H), 5.95 (br, 1H), 3.46 (q, 2H), $1.61(\mathrm{~m}, 2 \mathrm{H}), 1.25$ (s, 22H), 0.86 (t, 3H); EI-MS m/z $374\left(\mathrm{M}^{+}\right)$. Anal. Calcd for $\mathrm{C}_{21} \mathrm{H}_{34} \mathrm{~N}_{4} \mathrm{O}_{2}$ : C, 67.34; H, 9.15; N, 14.95. Found: C, 67.53; H, 9.51; N, 14.67.

1-Hydroxy-1H-benzo[d][1,2,3]triazole-6-carboxylic acid (4). To a solution of 4-chloro-3-nitrobenzoic acid $(2.51 \mathrm{~g}, 12.5 \mathrm{mmol})$ in dry $\mathrm{EtOH}(20 \mathrm{~mL})$ hydrazine hydrate $(6.2 \mathrm{~mL}, 125 \mathrm{mmol})$ was added. The mixture was heated to reflux for $15 \mathrm{~h}$. After that reaction was allowed to cool to room temperature, then acidified with conc. $\mathrm{HCl}$. During acidification, a white solid started precipitating out. After acidification and cooling, the solid was filtered and washed with hot EtOH. Then the residue was recrystallized using dry EtOH to afford 4 as a white solid $(1.7 \mathrm{~g}, 76 \%)$. mp above $70{ }^{\circ} \mathrm{C}$ decomposes; IR (KBr), $v_{\max } 3320$, 1700, $840 \mathrm{~cm}^{-1}$; ${ }^{1} \mathrm{H}-\mathrm{NMR}\left(\mathrm{D}_{2} \mathrm{O}, 300 \mathrm{MHz}\right) \delta 8.15$ (s, 1H), 7.87 (dd, 1H), 7.65 (d, 1H); EI-MS; m/z 179 $\left(\mathrm{M}^{+}\right)$. Anal. Calcd for $\mathrm{C}_{7} \mathrm{H}_{5} \mathrm{~N}_{3} \mathrm{O}_{3}: \mathrm{C}, 46.93 ; \mathrm{H}, 2.81 ; \mathrm{N}, 23.45$. Found: C, 46.9; H, 2.78; N, 23.43.

1-Hydroxy-1H-benzo[d][1,2,3]triazole-7-carboxylic acid (5). To a solution of 3-chloro-2-nitrobenzoic acid $(0.31 \mathrm{~g}, 1.5 \mathrm{mmol})$ in dry $\mathrm{EtOH}(5 \mathrm{~mL})$, hydrazine hydrate $(0.73 \mathrm{~mL}, 15 \mathrm{mmol})$ was added. The mixture was heated to reflux for $18 \mathrm{~h}$. After that reaction was allowed to cool to room temperature, then acidified with conc. HCl. During acidification, a white solid started precipitating out. The solid was filtered and washed with hot $\mathrm{EtOH}$. The residue was recrystallized from dry EtOH to afford $\mathbf{5}$ as a white solid $(0.17 \mathrm{~g}, 62 \%)$. mp above $70{ }^{\circ} \mathrm{C}$ decomposes; IR (KBr), $v_{\text {max }} 3315,1704,830 \mathrm{~cm}^{-1} ;{ }^{1} \mathrm{H}-\mathrm{NMR}\left(\mathrm{D}_{2} \mathrm{O}\right.$, $300 \mathrm{MHz}) \delta 8.14(\mathrm{~d}, 2 \mathrm{H}), 7.35(\mathrm{t}, 1 \mathrm{H}) ; \mathrm{EI}-\mathrm{MS} ; \mathrm{m} / \mathrm{z} 179\left(\mathrm{M}^{+}\right)$. Anal. Calcd for $\mathrm{C}_{7} \mathrm{H}_{5} \mathrm{~N}_{3} \mathrm{O}_{3}: \mathrm{C}, 46.93 ; \mathrm{H}$, $2.81 ; \mathrm{N}, 23.45$. Found: C, 46.92; H, 2.79; N, 23.42 . 
Table S1. Kinetic and Thermodynamic Parameters for the cleavage of PNPDPP by 1-5 with CTABr micelles. ${ }^{\text {a }}$

\begin{tabular}{llrl}
\hline catalyst & $10^{3} k_{\lim }, \mathrm{s}^{-1}$ & $\mathrm{~K}_{\mathrm{b}}, \mathrm{M}^{-1}$ & $10^{3} k_{2}, \mathrm{M}^{-1} \mathrm{~s}^{-1}$ \\
\hline $\mathbf{1}$ & 4.58 & 885 & 72.1 \\
$\mathbf{2}$ & 5.94 & 1081 & 89.7 \\
$\mathbf{3}$ & 5.8 & 926 & 88.6 \\
$\mathbf{4}$ & 6.72 & 1431 & 101.4 \\
$\mathbf{5}$ & 6.16 & 1250 & 94.1 \\
\hline
\end{tabular}

${ }^{a}$ Conditions: Kinetic cleavage experiments at $\mathrm{pH} 8.2,25{ }^{\circ} \mathrm{C}$ and $0.05 \mathrm{M}$ tris-maleate buffer using solutions containing increasing amounts of catalyst and $\mathrm{CTABr}$ with molar ratios 1:30, 1:40 and 1:50 using PNPDPP as substrate. ${ }^{\mathrm{b}} k_{\lim }$, and $k_{2}$ were calculated by fitting in Michaelis-Menten equation. 
Table S2a. Calculated bond lengths ( $\AA$ ) for most stable conformations of 1-13 at B3LYP/6-31+G* and RHF6-31+G* levels of theory. RHF values are shown in parentheses.

\begin{tabular}{|c|c|c|c|c|c|c|c|c|c|}
\hline acid & $\mathrm{N}_{1}-\mathrm{O}_{7}$ & $\mathrm{~N}_{1}-\mathrm{N}_{2}$ & $\mathrm{~N}_{2}-\mathrm{N}_{3}$ & $\mathrm{~N}_{3}-\mathrm{C}_{1}$ & $\mathrm{~N}_{1}-\mathrm{C}_{2}$ & $\mathrm{O}_{7}-\mathrm{H}_{8}$ & $\mathrm{C}_{1}-\mathrm{C}_{2}$ & $\mathrm{C}_{2}-\mathrm{C}_{3}$ & $\mathrm{C}_{3}-\mathrm{C}_{4}$ \\
\hline \multirow[t]{2}{*}{1} & 1.371 & 1.353 & 1.298 & 1.381 & 1.363 & 0.976 & 1.41 & 1.402 & 1.388 \\
\hline & (1.339) & (1.319) & $(1.258)$ & (1.372) & (1.350) & $(0.952)$ & (1.384) & (1.401) & $(1.370)$ \\
\hline \multirow[t]{2}{*}{2} & 1.371 & 1.352 & 1.298 & 1.381 & 1.363 & 0.976 & 1.409 & 1.40 & 1.393 \\
\hline & (1.339) & (1.317) & (1.259) & (1.371) & (1.351) & $(0.952)$ & (1.382) & (1.399) & (1.373) \\
\hline \multirow[t]{2}{*}{3} & 1.375 & 1.355 & 1.29 & 1.379 & 1.375 & 0.976 & 1.417 & 1.42 & 1.396 \\
\hline & (1.34) & (1.324) & $(1.252)$ & (1.370) & (1.359) & $(0.952)$ & (1.390) & (1.416) & (1.377) \\
\hline \multirow[t]{2}{*}{4} & 1.370 & 1.349 & 1.300 & 1.380 & 1.364 & 0.976 & 1.41 & 1.398 & 1.393 \\
\hline & (1.339) & (1.315) & $(1.260)$ & (1.371) & $(1.352)$ & $(0.952)$ & (1.383) & (1.397) & (1.374) \\
\hline \multirow[t]{2}{*}{5} & 1.368 & 1.345 & 1.301 & 1.372 & 1.360 & 0.996 & 1.416 & 1.422 & 1.398 \\
\hline & (1.344) & (1.316) & $(1.258)$ & (1.366) & (1.347) & $(0.957)$ & (1.388) & (1.413) & (1.378) \\
\hline \multirow[t]{2}{*}{6} & 1.369 & 1.349 & 1.299 & 1.379 & 1.363 & 0.976 & 1.411 & 1.397 & 1.386 \\
\hline & (1.338) & (1.314) & $(1.260)$ & (1.370) & $(1.351)$ & $(0.952)$ & (1.384) & (1.395) & (1.368) \\
\hline \multirow[t]{2}{*}{7} & 1.366 & 1.348 & 1.297 & 1.373 & 1.359 & 0.99 & 1.419 & 1.146 & 1.392 \\
\hline & (1.340) & (1.324) & $(1.252)$ & (1.371) & (1.349) & $(0.953)$ & (1.388) & (1.403) & (1.372) \\
\hline \multirow[t]{2}{*}{8} & 1.371 & 1.357 & 1.295 & 1.381 & 1.360 & 0.976 & 1.41 & 1.402 & 1.381 \\
\hline & (1.339) & (1.324) & $(1.255)$ & (1.372) & $(1.346)$ & $(0.952)$ & (1.384) & (1.401) & (1.362) \\
\hline \multirow[t]{2}{*}{9} & 1.369 & 1.354 & 1.294 & 1.382 & 1.364 & 0.976 & 1.41 & 1.399 & 1.378 \\
\hline & (1.338) & (1.318) & $(1.257)$ & (1.372) & $(1.351)$ & $(0.951)$ & (1.383) & (1.396) & (1.359) \\
\hline \multirow[t]{2}{*}{10} & 1.373 & 1.363 & 1.296 & 1.379 & 1.362 & 0.975 & 1.408 & 1.397 & 1.408 \\
\hline & (1.340) & (1.329) & $(1.256)$ & (1.370) & (1.347) & $(0.951)$ & (1.381) & (1.398) & (1.386) \\
\hline \multirow[t]{2}{*}{11} & 1.375 & 1.336 & 1.312 & 1.380 & 1.353 & 0.999 & 1.405 & 1.401 & 1.388 \\
\hline & (1.339) & (1.316) & $(1.258)$ & (1.370) & (1.356) & $(0.951)$ & (1.383) & (1.413) & (1.372) \\
\hline \multirow[t]{2}{*}{12} & 1.371 & 1.360 & 1.294 & 1.382 & 1.363 & 0.976 & 1.396 & 1.394 & 1.425 \\
\hline & (1.340) & (1.328) & $(1.254)$ & (1.374) & (1.348) & $(0.951)$ & (1.394) & (1.377) & (1.421) \\
\hline \multirow[t]{2}{*}{13} & 1.370 & 1.351 & 1.297 & 1.381 & 1.367 & 0.975 & 1.411 & 1.390 & 1.422 \\
\hline & (1.339) & (1.314) & $(1.260)$ & (1.369) & $(1.354)$ & $(0.951)$ & (1.416) & (1.372) & $(1.430)$ \\
\hline
\end{tabular}


Table S2b. Calculated bond lengths (A) for most stable conformations of conjugate bases of 1-13 at B3LYP/6-31+G* and RHF6-31+G* levels of theory. RHF values are shown in parentheses.

\begin{tabular}{|c|c|c|c|c|c|c|c|c|}
\hline base & $\mathrm{N}_{1}-\mathrm{O}_{7}$ & $\mathrm{~N}_{1}-\mathrm{N}_{2}$ & $\mathrm{~N}_{2}-\mathrm{N}_{3}$ & $\mathrm{~N}_{3}-\mathrm{C}_{1}$ & $\mathrm{~N}_{1}-\mathrm{C}_{2}$ & $\mathrm{C}_{1}-\mathrm{C}_{2}$ & $\mathrm{C}_{2}-\mathrm{C}_{3}$ & $\mathrm{C}_{3}-\mathrm{C}_{4}$ \\
\hline \multirow[t]{2}{*}{$1-0^{-}$} & 1.30 & 1.350 & 1.340 & 1.360 & 1.372 & 1.417 & 1.402 & 1.389 \\
\hline & (1.304) & (1.290) & $(1.305)$ & $(1.344)$ & $(1.347)$ & (1.384) & (1.401) & (1.370) \\
\hline \multirow[t]{2}{*}{$2-0^{-}$} & 1.295 & 1.345 & 1.346 & 1.354 & 1.376 & 1.417 & 1.392 & 1.398 \\
\hline & $(1.300)$ & $(1.284)$ & $(1.313)$ & (1.338) & $(1.350)$ & (1.392) & (1.395) & (1.375) \\
\hline \multirow[t]{2}{*}{ 3-O- } & 1.318 & 1.347 & 1.324 & 1.362 & 1.376 & 1.423 & 1.428 & 1.392 \\
\hline & $(1.315)$ & $(1.297)$ & $(1.287)$ & (1.347) & $(1.351)$ & (1.393) & $(1.430)$ & (1.371) \\
\hline \multirow[t]{2}{*}{$4-0^{-}$} & 1.310 & 1.347 & 1.345 & 1.363 & 1.379 & 1.415 & 1.408 & 1.393 \\
\hline & (1.308) & $(1.289)$ & $(1.309)$ & (1.346) & (1.354) & (1.390) & (1.411) & (1.371) \\
\hline \multirow[t]{2}{*}{$5-0^{-}$} & 1.295 & 1.348 & 1.345 & 1.359 & 1.389 & 1.428 & 1.418 & 1.397 \\
\hline & $(1.298)$ & (1.287) & $(1.311)$ & $(1.341)$ & $(1.362)$ & $(1.40)$ & $(1.420)$ & $(1.375)$ \\
\hline \multirow[t]{2}{*}{$6-\mathrm{O}^{-}$} & 1.285 & 1.341 & 1.351 & 1.346 & 1.385 & 1.421 & 1.385 & 1.397 \\
\hline & $(1.292)$ & $(1.279)$ & $(1.321)$ & (1.330) & $(1.356)$ & (1.397) & (1.388) & (1.373) \\
\hline \multirow[t]{2}{*}{$7-0^{-}$} & 1.285 & 1.367 & 1.326 & 1.360 & 1.369 & 1.425 & 1.411 & 1.389 \\
\hline & $(1.291)$ & $(1.298)$ & $(1.301)$ & (1.338) & $(1.344)$ & (1.398) & (1.406) & (1.361) \\
\hline \multirow[t]{2}{*}{ 8-O- } & 1.299 & 1.356 & 1.335 & 1.362 & 1.367 & 1.417 & 1.406 & 1.376 \\
\hline & $(1.305)$ & $(1.297)$ & $(1.298)$ & $(1.347)$ & $(1.341)$ & $(1.392)$ & (1.408) & (1.355) \\
\hline \multirow[t]{2}{*}{ 9-0 } & 1.293 & 1.351 & 1.338 & 1.356 & 1.376 & 1.422 & 1.401 & 1.380 \\
\hline & $(1.298)$ & $(1.287)$ & $(1.307)$ & (1.338) & $(1.351)$ & (1.383) & (1.396) & (1.359) \\
\hline \multirow[t]{2}{*}{$10-0^{-}$} & 1.300 & 1.355 & 1.377 & 1.361 & 1.368 & 1.412 & 1.405 & 1.391 \\
\hline & (1.305) & $(1.295)$ & $(1.302)$ & (1.346) & $(1.343)$ & (1.388) & (1.408) & (1.367) \\
\hline \multirow[t]{2}{*}{$11-0^{-}$} & 1.300 & 1.347 & 1.333 & 1.357 & 1.382 & 1.421 & 1.430 & 1.399 \\
\hline & (1.304) & (1.289) & $(1.302)$ & (1.338) & (1.357) & (1.383) & (1.413) & (1.372) \\
\hline \multirow[t]{2}{*}{$12-0^{-}$} & 1.301 & 1.355 & 1.335 & 1.364 & 1.369 & 1.401 & 1.387 & 1.425 \\
\hline & (1.307) & (1.298) & $(1.296)$ & (1.351) & $(1.342)$ & (1.394) & (1.377) & (1.421) \\
\hline \multirow[t]{2}{*}{ 13- $0^{-}$} & 1.294 & 1.351 & 1.339 & 1.355 & 1.378 & 1.414 & 1.393 & 1.428 \\
\hline & (1.298) & (1.287) & (1.309) & (1.336) & $(1.355)$ & (1.416) & (1.372) & (1.430) \\
\hline
\end{tabular}


Table S3a. Selected bond angles (in degrees) for most stable conformations of 1-13 at B3LYP/6-31+G* and RHF6-31+G* levels of theory. RHF values are shown in parentheses.

\begin{tabular}{|c|c|c|c|c|c|c|}
\hline acid & $\mathrm{O}_{7}-\mathrm{N}_{1}-\mathrm{N}_{2}$ & $\mathrm{O}_{7}-\mathrm{N}_{1}-\mathrm{C}_{2}$ & $\mathrm{C}_{2}-\mathrm{N}_{1}-\mathrm{N}_{2}$ & $\mathrm{~N}_{1}-\mathrm{N}_{2}-\mathrm{N}_{3}$ & $\mathrm{~N}_{1}-\mathrm{C}_{1}-\mathrm{C}_{2}$ & $\mathrm{~N}_{1}-\mathrm{O}_{7}-\mathrm{H}_{8}$ \\
\hline \multirow[t]{2}{*}{1} & 120.7 & 126.3 & 112.4 & 107.9 & 102.0 & 105.6 \\
\hline & (120.6) & (127.1) & (111.8) & (109.1) & (102.2) & (107.1) \\
\hline \multirow[t]{2}{*}{2} & 121.0 & 126.0 & 112.3 & 108.0 & 102.1 & 105.6 \\
\hline & (120.7) & (127.0) & (111.7) & (109.2) & (102.3) & (107.1) \\
\hline \multirow[t]{2}{*}{3} & 118.0 & 128.8 & 112.9 & 107.9 & 100.6 & 105.8 \\
\hline & (118.4) & (129.1) & (121.2) & (109.1) & (101.1) & (107.3) \\
\hline \multirow[t]{2}{*}{4} & 121.0 & 126.1 & 112.2 & 108.1 & 102.1 & 105.6 \\
\hline & (120.8) & (127.0) & (111.6) & (109.4) & (102.3) & (107.1) \\
\hline \multirow[t]{2}{*}{5} & 116.9 & 130.9 & 112.2 & 108.6 & 101.9 & 104.2 \\
\hline & $(117.3)$ & (129.8) & (111.5) & (109.6) & (102.2) & (106.1) \\
\hline \multirow[t]{2}{*}{6} & 121.2 & 126.0 & 112.1 & 108.3 & 102.2 & 105.8 \\
\hline & (120.9) & $(127.0)$ & (111.5) & (109.5) & (102.3) & (107.3) \\
\hline \multirow[t]{2}{*}{7} & 116.7 & 130.9 & 112.0 & 108.6 & 102.0 & 103.4 \\
\hline & (117.7) & (128.9) & (111.1) & (109.7) & (102.5) & (106.8) \\
\hline \multirow[t]{2}{*}{8} & 121.0 & 126.1 & 112.3 & 107.9 & 102.1 & 105.6 \\
\hline & (120.7) & (127.0) & (111.8) & (109.0) & (102.2) & (107.1) \\
\hline \multirow[t]{2}{*}{9} & 120.4 & 126.8 & 111.7 & 108.2 & 102.7 & 105.8 \\
\hline & (120.3) & (127.6) & (111.2) & (109.4) & (102.8) & (107.2) \\
\hline \multirow[t]{2}{*}{10} & 120.7 & 120.1 & 112.5 & 107.5 & 101.8 & 105.4 \\
\hline & (120.5) & (127.1) & (111.9) & (108.7) & (102.0) & (107.0) \\
\hline \multirow[t]{2}{*}{11} & 121.6 & 126.0 & 112.4 & 107.9 & 103.0 & 101.3 \\
\hline & (119.4) & (128.7) & (111.7) & (109.2) & $(102.0)$ & (107.1) \\
\hline \multirow[t]{2}{*}{12} & 120.7 & 126.2 & 112.3 & 107.4 & 101.9 & 105.6 \\
\hline & (120.4) & (127.1) & (111.8) & (109.0) & (102.0) & $(107.0)$ \\
\hline \multirow[t]{2}{*}{13} & 119.7 & 127.4 & 111.8 & 108.1 & 102.5 & 105.8 \\
\hline & (119.7) & (128.1) & (111.3) & (109.3) & (102.6) & (107.1) \\
\hline
\end{tabular}


Table S3b. Selected bond angles (in degrees) for most stable conformations of conjugate bases of 1-13 at B3LYP/6-31+G* and RHF6-31+G* levels of theory. RHF values are shown in parentheses.

\begin{tabular}{|c|c|c|c|c|c|}
\hline base & $\mathrm{O}_{7}-\mathrm{N}_{1}-\mathrm{N}_{2}$ & $\mathrm{O}_{7}-\mathrm{N}_{1}-\mathrm{C}_{2}$ & $\mathrm{C}_{2}-\mathrm{N}_{1}-\mathrm{N}_{2}$ & $\mathrm{~N}_{1}-\mathrm{N}_{2}-\mathrm{N}_{3}$ & $\mathrm{~N}_{1}-\mathrm{C}_{1}-\mathrm{C}_{2}$ \\
\hline \multirow[t]{2}{*}{$1-0^{-}$} & 123.9 & 126.8 & 109.3 & 109.8 & 104.5 \\
\hline & (124.1) & (126.1) & (109.8) & (110.9) & $(104.2)$ \\
\hline \multirow[t]{2}{*}{$2-0^{-}$} & 124.3 & 126.4 & 104.6 & 109.8 & 104.6 \\
\hline & $(124.5)$ & (125.7) & (109.7) & $(110.9)$ & (104.6) \\
\hline \multirow[t]{2}{*}{ 3-O- } & 120.8 & 128.9 & 110.3 & 109.8 & 102.9 \\
\hline & (120.9) & $(128.5)$ & (110.5) & (110.8) & (102.8) \\
\hline \multirow[t]{2}{*}{$4-0^{-}$} & 122.9 & 127.3 & 109.7 & 109.7 & 104.1 \\
\hline & (123.0) & (126.9) & (110.1) & (110.8) & (103.8) \\
\hline \multirow[t]{2}{*}{$5-0^{-}$} & 122.0 & 128.2 & 109.8 & 109.9 & 103.4 \\
\hline & (122.0) & (127.7) & $(110.2)$ & (111.1) & (103.0) \\
\hline \multirow[t]{2}{*}{ 6-O- } & 124.3 & 126.5 & 109.2 & 109.7 & 104.2 \\
\hline & (124.7) & (125.6) & (109.7) & (111.0) & (104.0) \\
\hline \multirow[t]{2}{*}{$7-0^{-}$} & 122.7 & 128.5 & 108.7 & 110.1 & 104.6 \\
\hline & (123.6) & $(127.0)$ & (109.3) & (111.2) & (104.3) \\
\hline \multirow[t]{2}{*}{$8-0^{-}$} & 123.9 & 126.9 & 109.2 & 109.8 & 104.7 \\
\hline & (124.0) & (126.2) & (109.8) & (110.9) & (104.3) \\
\hline \multirow[t]{2}{*}{ 9-O- } & 123.5 & 127.7 & 108.9 & 110.0 & 104.7 \\
\hline & (123.5) & (126.9) & (109.5) & (111.1) & (104.3) \\
\hline \multirow[t]{2}{*}{$10-0^{-}$} & 123.9 & 126.8 & 109.3 & 109.7 & 104.6 \\
\hline & $(124.0)$ & $(126.2)$ & (109.8) & $(110.8)$ & (104.3) \\
\hline \multirow[t]{2}{*}{$11-0^{-}$} & 121.7 & 128.5 & 109.7 & 109.8 & 103.5 \\
\hline & $(122.0)$ & $(127.9)$ & $(110.1)$ & $(110.9)$ & (103.3) \\
\hline \multirow[t]{2}{*}{$12-\mathrm{O}^{-}$} & 123.9 & 126.9 & 109.2 & 110.0 & 104.6 \\
\hline & (123.8) & (126.4) & (109.8) & $(111.0)$ & $(104.2)$ \\
\hline \multirow[t]{2}{*}{$13-\mathrm{O}^{-}$} & 122.8 & 128.1 & 109.0 & 109.9 & 104.5 \\
\hline & $(122.8)$ & $(127.5)$ & (109.7) & (110.9) & (103.9) \\
\hline
\end{tabular}


Table S4. Selected torsional angles (in degrees) for 1-13 and their conjugate bases at B3LYP/6-31+G* and RHF6-31+G* levels of theory. RHF values are shown in parentheses.

\begin{tabular}{|c|c|c|c|c|}
\hline acid & $\mathrm{N}_{1}-\mathrm{N}_{2}-\mathrm{N}_{3}-\mathrm{C}_{1}$ & $\mathrm{~N}_{2}-\mathrm{N}_{1}-\mathrm{O}_{7}-\mathrm{H}_{8}$ & conjugate base & $\mathrm{N}_{1}-\mathrm{N}_{2}-\mathrm{N}_{3}-\mathrm{C}$ \\
\hline \multirow[t]{2}{*}{1} & 1.4 & 70.3 & $1-0^{-}$ & 0.0 \\
\hline & (1.9) & $(81.0)$ & & $(0.0)$ \\
\hline \multirow[t]{2}{*}{2} & 1.8 & 69.4 & $2-\mathrm{O}^{-}$ & 0.2 \\
\hline & (1.3) & $(78.5)$ & & $(0.2)$ \\
\hline \multirow[t]{2}{*}{3} & 1.2 & 75.7 & $3-\mathrm{O}^{-}$ & 0.0 \\
\hline & (1.5) & $(85.7)$ & & $(0.0)$ \\
\hline \multirow[t]{2}{*}{4} & 1.67 & 71.56 & $4-0^{-}$ & 0.0 \\
\hline & (1.3) & (81.1) & & $(0.0)$ \\
\hline \multirow[t]{2}{*}{5} & 0.0 & 179.9 & $5-\mathrm{O}^{-}$ & 0.0 \\
\hline & $(0.87)$ & (148.7 & & $(0.0)$ \\
\hline \multirow[t]{2}{*}{6} & 1.74 & 69.8 & 6- $\mathrm{O}^{-}$ & 0.0 \\
\hline & (1.38) & (81.2) & & $(0.0)$ \\
\hline \multirow[t]{2}{*}{7} & 0.2 & 166.5 & $7-\mathrm{O}^{-}$ & 0.2 \\
\hline & (1.4) & (126.8 & & $(0.8)$ \\
\hline \multirow[t]{2}{*}{8} & 1.7 & 70.2 & 8- $\mathrm{O}^{-}$ & 0.0 \\
\hline & (1.3) & $(80.7)$ & & $(0.0)$ \\
\hline \multirow[t]{2}{*}{9} & 1.8 & 83.1 & $9-\mathrm{O}^{-}$ & 0.0 \\
\hline & (1.44) & $(89.1)$ & & $(0.0)$ \\
\hline \multirow[t]{2}{*}{10} & 1.17 & 71.3 & $10-0^{-}$ & 0.2 \\
\hline & $(1.23)$ & (81.1) & & $(0.1)$ \\
\hline \multirow[t]{2}{*}{11} & 0.0 & 179.9 & 11-O'- & 0.32 \\
\hline & $(0.54)$ & (97.0) & & $(0.5)$ \\
\hline \multirow[t]{2}{*}{12} & 1.8 & 73.6 & $12-\mathrm{O}^{-}$ & 0.07 \\
\hline & $(2.2)$ & $(83.0)$ & & $(0.06)$ \\
\hline \multirow[t]{2}{*}{13} & 1.5 & 88.9 & 13-O- & 0.04 \\
\hline & $(1.2)$ & (94.2) & & $(0.02)$ \\
\hline
\end{tabular}


Table S5. Total energies (in Hartree) for 1-13 and their conjugate bases at B3LYP/6-31+G* level.

\begin{tabular}{|c|c|c|c|}
\hline molecule & energy & conjugate base & energy \\
\hline 1 & -471.0432882 & $1-\mathrm{O}^{-}$ & -470.5162442 \\
\hline 2 & -679.0638614 & $2-\mathrm{O}^{-}$ & -678.5476959 \\
\hline 3 & -679.0624826 & $3-\mathrm{O}^{-}$ & -678.5598858 \\
\hline 4 & -659.6244116 & 4-O- & $-659.1114963^{a}(658.4661331)^{b}$ \\
\hline 5 & -659.6299036 & $5-\mathrm{O}^{-}$ & $-659.0946354^{a}(658.4385984)^{b}$ \\
\hline 6 & -675.5505697 & 6- $\mathrm{O}^{-}$ & -675.0489897 \\
\hline 7 & -675.5502544 & $7-\mathrm{O}^{-}$ & -675.0273956 \\
\hline 8 & -570.2838148 & 8- $\mathrm{O}^{-}$ & -569.7618852 \\
\hline 9 & -570.2778426 & 9-0- & -569.7546037 \\
\hline 10 & -605.0145191 & $10-\mathrm{O}^{-}$ & -604.4823216 \\
\hline 11 & -605.0099919 & $11-\mathrm{O}^{-}$ & -604.4817276 \\
\hline 12 & -585.5687875 & $11-0^{-}$ & -585.0375656 \\
\hline 13 & -585.5658729 & $13-\mathrm{O}^{-}$ & -585.0329644 \\
\hline
\end{tabular}

${ }^{a}$ Energy of carboxylic acid $\left(\mathrm{N}-\mathrm{O}^{-}\right)$form. ${ }^{b}$ Energy of di-deprotonated (carboxylate, $\mathrm{N}-\mathrm{O}^{-}$) form. 
Cartesian coordinates of full-optimized geometries of 1-13 and their conjugate bases at B3LYP/6$31+\mathrm{G}^{*}$.

\begin{tabular}{lrcc}
\multicolumn{4}{c}{1} \\
\\
7 & -1.866912 & 1.069748 & -0.013756 \\
7 & -1.508865 & -0.234612 & 0.018681 \\
6 & -0.155751 & -0.402045 & 0.006159 \\
6 & 0.305233 & 0.930605 & 0.002392 \\
7 & -0.782373 & 1.782934 & -0.001357 \\
6 & 1.684749 & 1.203307 & 0.002199 \\
6 & 2.546993 & 0.117455 & 0.001446 \\
6 & 2.062021 & -1.217575 & 0.001339 \\
6 & 0.705033 & -1.509374 & 0.004374 \\
8 & -2.448911 & -1.225829 & -0.106263 \\
1 & 2.045647 & 2.227282 & 0.000903 \\
1 & 3.620491 & 0.283909 & -0.000930 \\
1 & 2.777799 & -2.035185 & -0.002761 \\
1 & 0.330771 & -2.527690 & -0.001107 \\
1 & -2.966036 & -1.202417 & 0.721564
\end{tabular}

\section{1-0}

$\begin{array}{lll}0.893495 & -1.824754 & 0.000000\end{array}$

$\begin{array}{llll}2.163665 & -1.264697 & 0.000000\end{array}$

$2.359030 \quad 0.148461 \quad 0.000000$

$\begin{array}{lll}1.279555 & 1.022675 & 0.000000\end{array}$

$\begin{array}{lll}0.000000 & 0.448642 & 0.000000\end{array}$

$\begin{array}{lll}-0.217724 & -0.951961 & 0.000000\end{array}$

$\begin{array}{lll}-1.259466 & 0.993215 & 0.000000\end{array}$

$\begin{array}{lll}-1.557114 & -1.188524 & 0.000000\end{array}$

$\begin{array}{lll}-2.175635 & 0.001002 & 0.000000\end{array}$

$\begin{array}{llll}-1.560309 & 2.257974 & 0.000000\end{array}$

$\begin{array}{lll}0.747778 & -2.903323 & 0.000000\end{array}$

$\begin{array}{llll}3.037177 & -1.915348 & 0.000000\end{array}$

$\begin{array}{lll}3.373997 & 0.542307 & 0.000000\end{array}$

$\begin{array}{lll}1.400899 & 2.102522 & 0.000000\end{array}$

\section{2}

$\begin{array}{lll}-0.042138 & 0.603960 & 0.132341\end{array}$

$\begin{array}{lll}1.314911 & 0.270070 & 0.043628\end{array}$

$1.776098-1.051462-0.120093$

$\begin{array}{lll}0.856240 & -2.114123 & -0.182114\end{array}$

$\begin{array}{lll}-0.488030 & -1.800909 & -0.086420\end{array}$

$\begin{array}{lll}-0.941383 & -0.457716 & 0.054153\end{array}$

$\begin{array}{llll}3.155589 & -1.042022 & -0.178025\end{array}$

$\begin{array}{llll}3.565461 & 0.186093 & -0.073125\end{array}$

$\begin{array}{llll}2.480597 & 0.975795 & 0.093183\end{array}$

$\begin{array}{llll}2.596771 & 2.342780 & 0.104117\end{array}$

$1 \quad \begin{array}{llll}1.199452 & -3.137709 & -0.296288\end{array}$ 


$\begin{array}{lrrc}1 & -1.240575 & -2.581898 & -0.110800 \\ 1 & -0.359995 & 1.629489 & 0.288983 \\ 1 & 3.064274 & 2.559216 & 0.933696 \\ 6 & -2.430478 & -0.248141 & 0.152969 \\ 8 & -3.172346 & -1.110971 & 0.620090 \\ 7 & -2.905080 & 0.954709 & -0.291110 \\ 1 & -2.306075 & 1.523797 & -0.872613 \\ 6 & -4.333606 & 1.242934 & -0.287054 \\ 1 & -4.478318 & 2.300948 & -0.519436 \\ 1 & -4.873943 & 0.631262 & -1.020298 \\ 1 & -4.745877 & 1.030720 & 0.702183\end{array}$

2-0

$\begin{array}{lll}-3.568805 & -0.337370 & 0.044123\end{array}$

$\begin{array}{lll}-2.469498 & -1.106797 & -0.054630\end{array}$

$\begin{array}{lll}-1.351287 & -0.304452 & -0.033387\end{array}$

$\begin{array}{lll}-1.860168 & 1.013785 & 0.081427\end{array}$

$\begin{array}{lll}-3.212491 & 0.958966 & 0.125197\end{array}$

$\begin{array}{lll}-0.946706 & 2.094758 & 0.117256\end{array}$

$\begin{array}{lll}0.405287 & 1.813737 & 0.047405\end{array}$

$\begin{array}{llll}0.903134 & 0.473557 & -0.053361\end{array}$

$\begin{array}{llll}0.008003 & -0.600179 & -0.104630\end{array}$

$\begin{array}{lll}-2.487754 & -2.399272 & -0.144440\end{array}$

$\begin{array}{lll}-1.305740 & 3.118503 & 0.197625\end{array}$

$\begin{array}{lll}1.137483 & 2.615100 & 0.067591\end{array}$

$\begin{array}{llll}2.379951 & 0.291172 & -0.101782\end{array}$

$\begin{array}{llll}0.316989 & -1.634282 & -0.232384\end{array}$

$\begin{array}{lll}2.858598 & -0.976471 & 0.150236\end{array}$

$\begin{array}{lll}3.171096 & 1.210137 & -0.354706\end{array}$

$\begin{array}{lll}4.283052 & -1.242250 & 0.200469\end{array}$

$\begin{array}{llll}2.232671 & -1.648747 & 0.568332\end{array}$

$\begin{array}{llll}4.438394 & -2.324990 & 0.243065\end{array}$

$\begin{array}{lll}4.765629 & -0.846134 & -0.697595\end{array}$

$\begin{array}{lll}4.765614 & -0.775430 & 1.071670\end{array}$

\section{3}

$\begin{array}{lll}-0.275574 & 0.814670 & 0.032450\end{array}$

$\begin{array}{lll}0.723981 & -0.194818 & 0.014341\end{array}$

$\begin{array}{llll}2.103586 & 0.127130 & -0.045979\end{array}$

$\begin{array}{llll}2.551518 & 1.455676 & -0.089377\end{array}$

$\begin{array}{llll}1.586119 & 2.446875 & -0.063092\end{array}$

$\begin{array}{llll}0.214071 & 2.121528 & -0.001166\end{array}$

$\begin{array}{llll}2.854455 & -1.030319 & -0.039723\end{array}$

$\begin{array}{lll}2.054421 & -2.041742 & 0.013707\end{array}$

$\begin{array}{llll}0.785561 & -1.567397 & 0.069377\end{array}$ 


\begin{tabular}{lrrr}
8 & -0.245407 & -2.477235 & 0.054214 \\
1 & 3.614177 & 1.672249 & -0.137202 \\
1 & 1.876837 & 3.492834 & -0.091767 \\
6 & -1.785044 & 0.690587 & 0.071307 \\
1 & -0.264248 & -2.872545 & 0.947274 \\
1 & -0.527517 & 2.913201 & 0.022345 \\
8 & -2.479038 & 1.685295 & 0.293098 \\
7 & -2.347500 & -0.520286 & -0.173957 \\
1 & -1.766155 & -1.327635 & -0.351209 \\
6 & -3.795562 & -0.673955 & -0.193922 \\
1 & -4.029413 & -1.721478 & -0.399010 \\
1 & -4.231465 & -0.384493 & 0.768015 \\
1 & -4.243796 & -0.044553 & -0.970140 \\
& & & \\
& $\mathbf{3}-0$ & & \\
7 & 1.908529 & -2.129378 & -0.000158 \\
7 & 0.662264 & -1.615929 & 0.000110 \\
6 & 0.712173 & -0.240429 & 0.000059 \\
6 & 2.110367 & 0.028143 & -0.000193 \\
7 & 2.797614 & -1.148040 & -0.000332 \\
6 & 2.600174 & 1.347433 & -0.000192 \\
6 & 1.675193 & 2.378136 & 0.000102 \\
6 & 0.284053 & 2.109703 & 0.000337 \\
6 & -0.244318 & 0.821200 & 0.000259 \\
8 & -0.393254 & -2.406051 & 0.000492 \\
1 & 3.672364 & 1.529994 & -0.000392 \\
1 & 2.011483 & 3.413183 & 0.000139 \\
1 & -0.428350 & 2.928718 & 0.000555 \\
6 & -1.754398 & 0.723830 & 0.000287 \\
7 & -2.288801 & -0.513769 & -0.001082 \\
8 & -2.458870 & 1.750716 & 0.001146 \\
6 & -3.723245 & -0.698181 & -0.000741 \\
1 & -1.649141 & -1.342573 & -0.000624 \\
1 & -3.931184 & -1.772507 & -0.003904 \\
1 & -4.187304 & -0.244407 & 0.885493 \\
1 & -4.188117 & -0.238931 & -0.883657 \\
& & & \\
6 & 4 & & \\
6 & -0.297315 & -0.814266 & 0.005122 \\
6 & 0.978158 & -0.245447 & 0.006219 \\
6 & 1.204082 & 1.147021 & 0.004112 \\
6 & 0.116683 & 2.038730 & 0.003706 \\
6 & -1.155960 & 1.494342 & 0.002110 \\
6 & -1.362802 & 0.083853 & 0.002336 \\
7 & 2.564077 & 1.381473 & 0.000880 \\
7 & 3.179183 & 0.236476 & -0.011815 \\
7 & 2.249929 & -0.741412 & 0.020558 \\
1 & 2.605985 & -2.057638 & -0.115316 \\
& 0.280207 & 3.111786 & 0.002917 \\
1 & -2.022880 & 2.144703 & -0.000426 \\
& -0.469880 & -1.884564 & 0.001064 \\
\hline
\end{tabular}




$\begin{array}{rrrr}1 & 3.058590 & -2.296537 & 0.716492 \\ 6 & -2.734029 & -0.497637 & 0.001272 \\ 8 & -3.712815 & 0.443508 & -0.011795 \\ 8 & -2.984258 & -1.687813 & 0.011518 \\ 1 & -4.562561 & -0.035176 & -0.011924\end{array}$

\begin{tabular}{|c|c|c|c|}
\hline \multicolumn{4}{|c|}{$4-\mathrm{O}^{-}$} \\
\hline 7 & -2.917315 & -1.271560 & 0.000000 \\
\hline 7 & -2.393771 & -0.030459 & 0.000000 \\
\hline 6 & -1.016049 & -0.106465 & 0.000000 \\
\hline 6 & -0.748221 & -1.495980 & 0.000000 \\
\hline 7 & -1.926454 & -2.181938 & 0.000000 \\
\hline 6 & 0.599879 & -1.930832 & 0.000000 \\
\hline 6 & 1.598744 & -0.969757 & 0.000000 \\
\hline 6 & 1.323833 & 0.434838 & 0.000000 \\
\hline 6 & 0.000000 & 0.868708 & 0.000000 \\
\hline 8 & -3.128398 & 1.051290 & 0.000000 \\
\hline 1 & 0.834144 & -2.996058 & 0.000000 \\
\hline 1 & 2.647360 & -1.255215 & 0.000000 \\
\hline 6 & 2.499136 & 1.440969 & 0.000000 \\
\hline 1 & -0.223227 & 1.930436 & 0.000000 \\
\hline 8 & 2.197036 & 2.667720 & 0.000000 \\
\hline 8 & 3.663933 & 0.938446 & 0.000000 \\
\hline \multicolumn{4}{|c|}{5} \\
\hline 6 & -0.819114 & 0.501732 & -0.000458 \\
\hline 6 & 0.456528 & -0.126882 & -0.000440 \\
\hline 6 & 1.651266 & 0.634988 & 0.000255 \\
\hline 6 & 1.615889 & 2.037037 & 0.000434 \\
\hline 6 & 0.372798 & 2.656402 & -0.000187 \\
\hline 6 & -0.817251 & 1.900425 & -0.000624 \\
\hline 7 & 2.728084 & -0.216339 & 0.000896 \\
\hline 7 & 2.279617 & -1.437521 & 0.000015 \\
\hline 7 & 0.934506 & -1.400075 & -0.000136 \\
\hline 8 & 0.281096 & -2.602938 & -0.002082 \\
\hline 1 & 2.541668 & 2.604555 & 0.000977 \\
\hline 1 & 0.306086 & 3.739965 & -0.000043 \\
\hline 6 & -2.069226 & -0.267393 & 0.000608 \\
\hline 1 & -0.684701 & -2.356074 & 0.000967 \\
\hline 1 & -1.771626 & 2.415352 & -0.000653 \\
\hline 8 & -3.185381 & 0.484997 & -0.001087 \\
\hline 8 & -2.144052 & -1.496739 & 0.002644 \\
\hline 1 & -3.945530 & -0.126659 & -0.000008 \\
\hline
\end{tabular}




\begin{tabular}{|c|c|c|c|}
\hline \multicolumn{4}{|c|}{$5-0^{-}$} \\
\hline 6 & -1.699386 & 1.919589 & 0.000031 \\
\hline 6 & -0.479893 & 2.579562 & -0.000050 \\
\hline 6 & 0.750015 & 1.867451 & -0.000109 \\
\hline 6 & 0.827583 & 0.471659 & -0.000021 \\
\hline 6 & -0.425877 & -0.193242 & 0.000049 \\
\hline 6 & 2.241213 & -0.154609 & 0.000058 \\
\hline 6 & -1.667932 & 0.505030 & 0.000064 \\
\hline 7 & -0.807367 & -1.527747 & 0.000026 \\
\hline 7 & -2.694457 & -0.385566 & 0.000155 \\
\hline 7 & -2.152554 & -1.617170 & -0.000099 \\
\hline 8 & -0.048784 & -2.577977 & -0.000249 \\
\hline 8 & 2.757730 & -0.323933 & -1.136695 \\
\hline 1 & -2.651172 & 2.450692 & 0.000061 \\
\hline 1 & -0.454889 & 3.671709 & -0.000084 \\
\hline 1 & 1.682260 & 2.430761 & -0.000196 \\
\hline 8 & 2.757317 & -0.324642 & 1.136884 \\
\hline \multicolumn{4}{|c|}{6} \\
\hline 6 & -0.333086 & -0.818891 & 0.005368 \\
\hline 6 & 0.939615 & -0.242598 & 0.006985 \\
\hline 6 & 1.161663 & 1.151243 & 0.003749 \\
\hline 6 & 0.073851 & 2.042992 & 0.002196 \\
\hline 6 & -1.199584 & 1.501118 & 0.000493 \\
\hline 6 & -1.375925 & 0.095416 & 0.001901 \\
\hline 7 & 2.520406 & 1.387731 & 0.001182 \\
\hline 7 & 3.137155 & 0.244100 & -0.011580 \\
\hline 7 & 2.210840 & -0.736459 & 0.021610 \\
\hline 8 & 2.565911 & -2.051959 & -0.113813 \\
\hline 1 & 0.235594 & 3.115997 & 0.000585 \\
\hline 1 & -2.078891 & 2.133065 & -0.002980 \\
\hline 1 & -0.510031 & -1.886910 & 0.001781 \\
\hline 1 & 3.046750 & -2.282871 & 0.704675 \\
\hline 7 & -2.754505 & -0.435350 & 0.000156 \\
\hline 8 & -3.679944 & 0.376297 & -0.009144 \\
\hline 8 & -2.897205 & -1.658726 & 0.009483 \\
\hline \multicolumn{4}{|c|}{$6-\mathrm{O}^{-}$} \\
\hline 6 & 0.000000 & 0.873426 & 0.000000 \\
\hline 6 & 1.301893 & 0.364650 & 0.000000 \\
\hline 6 & 1.575871 & -1.039664 & 0.000000 \\
\hline 6 & 0.546668 & -1.957101 & 0.000000 \\
\hline 6 & -0.787681 & -1.475448 & 0.000000 \\
\hline 6 & -1.013395 & -0.071518 & 0.000000 \\
\hline 7 & 2.408430 & 1.287559 & 0.000000 \\
\hline 7 & -1.974340 & -2.111050 & 0.000000 \\
\hline 7 & -2.393820 & 0.051103 & 0.000000 \\
\hline 8 & 2.176750 & 2.510547 & 0.000000 \\
\hline
\end{tabular}




$\begin{array}{rrrc}8 & 3.566936 & 0.826992 & 0.000000 \\ 7 & -2.946262 & -1.171341 & 0.000000 \\ 8 & -3.063312 & 1.148207 & 0.000000 \\ 1 & -0.199077 & 1.938344 & 0.000000 \\ 1 & 2.610887 & -1.358795 & 0.000000 \\ 1 & 0.747010 & -3.025492 & 0.000000\end{array}$

\begin{tabular}{|c|c|c|c|}
\hline \multicolumn{4}{|c|}{7} \\
\hline 6 & -0.865882 & 0.466946 & -0.023305 \\
\hline 6 & 0.421067 & -0.125186 & -0.013340 \\
\hline 6 & 1.570232 & 0.707825 & 0.026224 \\
\hline 6 & 1.466847 & 2.105091 & 0.031331 \\
\hline 6 & 0.196268 & 2.667278 & -0.007687 \\
\hline 6 & -0.953684 & 1.856928 & -0.034182 \\
\hline 7 & 2.693260 & -0.082180 & 0.039758 \\
\hline 7 & 2.319885 & -1.324055 & 0.009737 \\
\hline 7 & 0.972435 & -1.367946 & -0.018692 \\
\hline 8 & 0.400598 & -2.600292 & -0.168224 \\
\hline 1 & 2.364744 & 2.714482 & 0.063536 \\
\hline 1 & 0.076542 & 3.745788 & -0.007144 \\
\hline 7 & -2.093713 & -0.303697 & 0.025778 \\
\hline 1 & -0.549884 & -2.442120 & 0.059215 \\
\hline 1 & -1.940410 & 2.305203 & -0.046380 \\
\hline 8 & -3.163697 & 0.273748 & -0.125662 \\
\hline 8 & -2.012293 & -1.529894 & 0.251444 \\
\hline \multicolumn{4}{|c|}{$7-0^{-}$} \\
\hline 6 & -0.825207 & 0.460910 & -0.03444 \\
\hline 6 & 0.429855 & -0.183267 & -0.003837 \\
\hline 6 & 1.620307 & 0.597094 & 0.063618 \\
\hline 6 & 1.565040 & 1.999566 & 0.092045 \\
\hline 6 & 0.319402 & 2.613828 & 0.023160 \\
\hline 6 & -0.869572 & 1.849300 & -0.048688 \\
\hline 1 & 2.483308 & 2.578863 & 0.154807 \\
\hline 1 & 0.244115 & 3.698452 & 0.040656 \\
\hline 1 & -1.837852 & 2.337589 & -0.07535 \\
\hline 7 & 2.693875 & -0.238747 & 0.043882 \\
\hline 7 & 0.870207 & -1.475486 & -0.11207 \\
\hline 7 & 2.237078 & -1.479555 & -0.05909 \\
\hline 8 & 0.178276 & -2.543336 & -0.29595 \\
\hline 7 & -2.093722 & -0.250530 & 0.07251 \\
\hline 8 & -3.054913 & 0.173566 & -0.59675 \\
\hline 8 & -2.158435 & -1.196387 & 0.85672 \\
\hline
\end{tabular}

\begin{tabular}{|c|c|c|c|}
\hline \multicolumn{4}{|c|}{8} \\
\hline 6 & -0.749357 & -1.117881 & 0.006644 \\
\hline 6 & 0.389636 & -0.299948 & 0.007296 \\
\hline 6 & 0.333273 & 1.109728 & 0.002605 \\
\hline & -0.909069 & 1.769143 & 0.001149 \\
\hline & -2.051567 & 0.986012 & 0.000556 \\
\hline
\end{tabular}




\begin{tabular}{|c|c|c|c|}
\hline 6 & -1.943024 & -0.422879 & 0.002225 \\
\hline 7 & 1.620223 & 1.610684 & -0.001989 \\
\hline 7 & 2.452504 & 0.617523 & -0.013124 \\
\hline 7 & 1.730442 & -0.532076 & 0.023154 \\
\hline 8 & 2.340846 & -1.752641 & -0.110155 \\
\hline 1 & -0.961395 & 2.853296 & -0.001187 \\
\hline 1 & -3.042858 & 1.427094 & -0.003307 \\
\hline 1 & -0.714156 & -2.200960 & 0.00225 \\
\hline 1 & 2.846199 & -1.884897 & 0.714866 \\
\hline 9 & -3.099565 & -1.132375 & -0.001170 \\
\hline \multicolumn{4}{|c|}{ 8-O- } \\
\hline 6 & 0.734073 & -1.100105 & -0.00000 \\
\hline 6 & -0.435546 & -0.319201 & -0.00002 \\
\hline 6 & -0.423302 & 1.098205 & 0.000002 \\
\hline 6 & 0.810839 & 1.781875 & 0.000037 \\
\hline 6 & 1.979529 & 1.031443 & 0.000044 \\
\hline 6 & 1.908487 & -0.382473 & 0.000027 \\
\hline 1 & 0.707180 & -2.184565 & -0.000023 \\
\hline 1 & 0.842105 & 2.869144 & 0.000028 \\
\hline 1 & 2.958244 & 1.503279 & 0.000048 \\
\hline 7 & -1.761092 & -0.655312 & -0.000058 \\
\hline 7 & -1.709625 & 1.546896 & -0.000096 \\
\hline 7 & -2.508353 & 0.476616 & 0.000100 \\
\hline 8 & -2.261310 & -1.854869 & -0.000041 \\
\hline 9 & 3.110218 & -1.064974 & 0.000015 \\
\hline \multicolumn{4}{|c|}{9} \\
\hline 6 & 0.792552 & 1.138053 & 0.004287 \\
\hline 6 & -0.183729 & 0.134582 & 0.003675 \\
\hline 6 & 0.184231 & -1.226762 & 0.004657 \\
\hline 6 & 1.536177 & -1.614557 & -0.001773 \\
\hline 6 & 2.485716 & -0.605479 & -0.01029 \\
\hline 6 & 2.120157 & 0.766152 & -0.007434 \\
\hline 7 & -0.968687 & -1.990466 & 0.00877 \\
\hline 7 & -1.995035 & -1.201383 & 0.001317 \\
\hline 7 & -1.547174 & 0.077397 & 0.033104 \\
\hline 8 & -2.417327 & 1.120319 & -0.142908 \\
\hline 1 & 1.809184 & -2.664667 & -0.000457 \\
\hline 1 & 3.542011 & -0.857189 & -0.018120 \\
\hline 1 & -2.822037 & 1.280031 & 0.731157 \\
\hline 1 & 2.879565 & 1.541886 & -0.012018 \\
\hline 9 & 0.432839 & 2.442957 & 0.020181 \\
\hline \multicolumn{4}{|c|}{ 9-O- } \\
\hline 6 & -0.809327 & 1.093353 & 0.000002 \\
\hline 6 & 0.232713 & 0.155529 & -0.000004 \\
\hline 6 & -0.065864 & -1.235394 & 0.00000 \\
\hline 6 & -1.407638 & -1.685232 & 0.00002 \\
\hline 6 & -2.412800 & -0.733885 & 0.00003 \\
\hline 6 & -2.120801 & 0.662125 & 0.00002 \\
\hline
\end{tabular}




$\begin{array}{rrrr}9 & -0.555054 & 2.431291 & -0.000010 \\ 1 & -1.627422 & -2.750096 & 0.000037 \\ 1 & -3.455829 & -1.044928 & 0.000047 \\ 7 & 1.607480 & 0.217968 & -0.000022 \\ 7 & 1.101103 & -1.926661 & -0.000003 \\ 7 & 2.103375 & -1.039169 & -0.000013 \\ 8 & 2.351977 & 1.276229 & -0.000036 \\ 1 & -2.918471 & 1.399626 & 0.000024\end{array}$

\begin{tabular}{lrrr}
\multicolumn{4}{c}{$\mathbf{1 0}$} \\
6 & -0.243145 & 0.783565 & -0.039388 \\
6 & 1.038299 & 0.225237 & -0.021261 \\
6 & 1.298057 & -1.158413 & -0.007862 \\
6 & 0.214791 & -2.053675 & -0.019793 \\
6 & -1.064302 & -1.532563 & -0.043287 \\
6 & -1.326109 & -0.116033 & -0.060627 \\
7 & 2.660845 & -1.368899 & 0.027114 \\
7 & 3.259310 & -0.218596 & 0.047965 \\
7 & 2.297670 & 0.745140 & -0.011993 \\
8 & 2.625170 & 2.071819 & 0.123339 \\
1 & 0.381551 & -3.126735 & -0.005587 \\
1 & -1.897832 & -2.223578 & -0.048317 \\
1 & -0.373495 & 1.857143 & -0.027374 \\
1 & 3.093661 & 2.309011 & -0.699412 \\
7 & -2.633079 & 0.344017 & -0.105808 \\
6 & -3.743836 & -0.574182 & 0.103970 \\
6 & -2.884275 & 1.771129 & 0.010581 \\
1 & -4.679465 & -0.015253 & 0.045117 \\
1 & -3.774518 & -1.348059 & -0.672479 \\
1 & -3.701927 & -1.070355 & 1.086223 \\
1 & -3.955806 & 1.956466 & -0.083027 \\
1 & -2.543826 & 2.181443 & 0.975400 \\
1 & -2.379808 & 2.323335 & -0.792190
\end{tabular}

\begin{tabular}{lccc}
\multicolumn{4}{c}{$\mathbf{1 0 - O}^{-}$} \\
6 & 0.372995 & 2.058212 & -0.199351 \\
6 & -0.936903 & 1.612903 & -0.254717 \\
6 & -1.277873 & 0.222207 & -0.164470 \\
6 & -0.272965 & -0.731221 & -0.036297 \\
6 & 1.053262 & -0.266651 & -0.002563 \\
6 & 1.402603 & 1.100778 & -0.066134 \\
7 & 2.248548 & -0.924707 & 0.106661 \\
7 & 2.757525 & 1.215559 & 0.003485 \\
7 & 3.258021 & -0.020658 & 0.110529 \\
8 & 2.425103 & -2.210477 & 0.200269 \\
7 & -2.672554 & -0.120248 & -0.247858 \\
6 & -3.480045 & 0.369078 & 0.866549 \\
6 & -2.970790 & -1.511494 & -0.538685 \\
1 & 0.608299 & 3.118065 & -0.277189 \\
1 & -1.742793 & 2.328993 & -0.401504
\end{tabular}




$\begin{array}{cccc}1 & -0.466858 & -1.795818 & 0.036653 \\ 1 & -3.281107 & -0.185152 & 1.806291 \\ 1 & -4.545598 & 0.262759 & 0.622826 \\ 1 & -3.273332 & 1.425808 & 1.050242 \\ 1 & -2.406748 & -1.836789 & -1.417921 \\ 1 & -2.732596 & -2.199037 & 0.297484 \\ 1 & -4.042585 & -1.607505 & -0.754750\end{array}$

11

$\begin{array}{lll}0.683786 & 0.523732 & 0.000006\end{array}$

$\begin{array}{lll}-0.544575 & -0.151089 & 0.000001\end{array}$

$\begin{array}{lll}-1.806549 & 0.468522 & -0.000043\end{array}$

$\begin{array}{lll}-1.870380 & 1.875810 & 0.000103\end{array}$

$\begin{array}{lll}-0.666290 & 2.567711 & 0.000149\end{array}$

$\begin{array}{lll}0.596564 & 1.909016 & 0.000093\end{array}$

$\begin{array}{lll}-2.770650 & -0.515536 & -0.000090\end{array}$

$\begin{array}{llll}-2.171457 & -1.683817 & -0.000089\end{array}$

$\begin{array}{llll}-0.852496 & -1.469430 & 0.000045\end{array}$

$\begin{array}{llll}0.046573 & -2.510920 & -0.000361\end{array}$

$\begin{array}{lll}-2.825058 & 2.392747 & 0.000115\end{array}$

$\begin{array}{lll}-0.678114 & 3.654163 & 0.000237\end{array}$

$\begin{array}{llll}1.884721 & -0.288679 & -0.000131\end{array}$

$\begin{array}{llll}0.916414 & -2.018794 & -0.000428\end{array}$

$\begin{array}{llll}1.501307 & 2.511378 & 0.000114\end{array}$

$\begin{array}{llll}2.693161 & -0.116364 & -1.219712\end{array}$

$\begin{array}{lll}2.692600 & -0.117567 & 1.219988\end{array}$

$\begin{array}{llll}3.497787 & -0.859362 & -1.220975\end{array}$

$\begin{array}{llll}2.065185 & -0.279328 & -2.099743\end{array}$

$\begin{array}{llll}3.144184 & 0.886723 & -1.289411\end{array}$

$\begin{array}{lll}3.497589 & -0.860169 & 1.220584\end{array}$

$\begin{array}{lll}3.143041 & 0.885661 & 1.291175\end{array}$

$\begin{array}{llll}2.064356 & -0.282048 & 2.099563\end{array}$

\section{1-0}

$\begin{array}{lll}0.773541 & 0.444536 & -0.065346\end{array}$

$\begin{array}{lll}0.784337 & 1.842778 & 0.003616\end{array}$

$\begin{array}{lll}-0.405480 & 2.620776 & 0.089359\end{array}$

$\begin{array}{lll}-1.653535 & 2.034778 & 0.131712\end{array}$

$\begin{array}{lll}-1.705611 & 0.622776 & 0.065029\end{array}$

$-0.522746-0.158960-0.042473$

$1.965453-0.305414 \quad-0.184446$

$\begin{array}{lll}-2.787867 & -0.195624 & 0.039971\end{array}$

$\begin{array}{llll}-0.999824 & -1.452394 & -0.146184\end{array}$

$\begin{array}{lll}-2.346157 & -1.447655 & -0.083743\end{array}$

$\begin{array}{lll}-0.324149 & -2.558845 & -0.290176\end{array}$

$\begin{array}{lll}2.160929 & -1.381770 & 0.787615\end{array}$

$\begin{array}{llll}3.183851 & 0.430826 & -0.449496\end{array}$

$\begin{array}{lll}1.732132 & 2.370186 & 0.009140\end{array}$

$\begin{array}{lll}-0.308998 & 3.704842 & 0.134758\end{array}$

$\begin{array}{lll}-2.569962 & 2.615934 & 0.199520\end{array}$

$\begin{array}{llll}1.271115 & -2.017200 & 0.809123\end{array}$ 


$\begin{array}{rrrc}1 & 2.389037 & -0.980690 & 1.795755 \\ 1 & 3.007535 & -1.999669 & 0.462407 \\ 1 & 3.048034 & 1.101023 & -1.304832 \\ 1 & 3.535876 & 1.036007 & 0.412299 \\ 1 & 3.975475 & -0.286497 & -0.696036\end{array}$

12

$\begin{array}{lrrr}6 & -0.389150 & -1.021313 & 0.011261 \\ 6 & 0.800853 & -0.291301 & 0.010240 \\ 6 & 0.850572 & 1.118747 & 0.004020 \\ 6 & -0.343155 & 1.854786 & 0.003436 \\ 6 & -1.541121 & 1.155032 & 0.004701 \\ 6 & -1.564395 & -0.269899 & 0.006963 \\ 7 & 2.171255 & 1.526128 & -0.005627 \\ 7 & 2.929282 & 0.476490 & -0.016792 \\ 7 & 2.123605 & -0.620012 & 0.025297 \\ 8 & 2.647002 & -1.880192 & -0.115740 \\ 1 & -0.325220 & 2.940442 & 0.000158 \\ 1 & -2.472426 & 1.708818 & 0.001788 \\ 1 & -0.421774 & -2.104761 & 0.006689 \\ 1 & 3.106341 & -2.069005 & 0.724703 \\ 8 & -2.720488 & -0.995919 & 0.005198 \\ 6 & -3.972660 & -0.319358 & -0.011144 \\ 1 & -4.728810 & -1.106030 & -0.015118 \\ 1 & -4.101940 & 0.304947 & 0.882085 \\ 1 & -4.082946 & 0.296069 & -0.912966\end{array}$

\section{2-O-}

$\begin{array}{lll}2.969712 & 0.355252 & 0.001424\end{array}$

$\begin{array}{lll}2.239142 & 1.473144 & 0.001831\end{array}$

$\begin{array}{lll}0.926725 & 1.100326 & -0.000182\end{array}$

$\begin{array}{llll}0.851413 & -0.314443 & -0.000592\end{array}$

$\begin{array}{lll}2.156236 & -0.729438 & 0.000955\end{array}$

$\begin{array}{lll}-0.359920 & -1.020025 & -0.001840\end{array}$

$\begin{array}{llll}-1.521458 & -0.260781 & -0.003061\end{array}$

$\begin{array}{llll}-1.480177 & 1.164544 & -0.002847\end{array}$

$\begin{array}{llll}-0.266047 & 1.845482 & -0.001333\end{array}$

$\begin{array}{lll}-2.716252 & -0.978376 & -0.005644\end{array}$

$\begin{array}{lll}-3.930146 & -0.263185 & 0.006997\end{array}$

$\begin{array}{llll}2.585123 & -1.958187 & 0.001091\end{array}$

$-0.389494-2.104860-0.001884$

$\begin{array}{llll}-2.402287 & 1.736764 & -0.004007\end{array}$

$\begin{array}{llll}-0.240636 & 2.933409 & -0.000959\end{array}$

$\begin{array}{lll}-4.724271 & -1.015891 & 0.009235\end{array}$

$\begin{array}{llll}-4.043086 & 0.373742 & -0.883829\end{array}$

$\begin{array}{lll}-4.029164 & 0.365115 & 0.905544\end{array}$

13

6

6

$\begin{array}{lll}-1.047799 & 0.056815 & 0.004715\end{array}$

$\begin{array}{lll}0.353033 & -0.119270 & 0.001884\end{array}$

6

$\begin{array}{llll}1.248370 & 0.965458 & 0.005414\end{array}$ 


$\begin{array}{lrrc}6 & 0.780948 & 2.294362 & -0.000868 \\ 6 & -0.590352 & 2.469038 & -0.011873 \\ 6 & -1.496313 & 1.372955 & -0.008666 \\ 7 & 2.539413 & 0.473064 & 0.011235 \\ 7 & 2.492905 & -0.823132 & 0.004155 \\ 7 & 1.194245 & -1.197754 & 0.028435 \\ 8 & 0.863734 & -2.515902 & -0.145250 \\ 1 & 1.474797 & 3.128346 & 0.001803 \\ 1 & -1.005857 & 3.472883 & -0.020250 \\ 8 & -1.820616 & -1.063614 & 0.027796 \\ 1 & 0.873039 & -2.912045 & 0.746510 \\ 1 & -2.560090 & 1.582361 & -0.013205 \\ 6 & -3.235185 & -0.899742 & -0.000408 \\ 1 & -3.648310 & -1.909338 & 0.010699 \\ 1 & -3.587354 & -0.347173 & 0.880058 \\ 1 & -3.553331 & -0.381856 & -0.913936\end{array}$

\section{3-O-}

6

$\begin{array}{lll}-1.018373 & 0.038601 & 0.000057\end{array}$

$\begin{array}{lll}-1.460145 & 1.359974 & 0.000046\end{array}$

$\begin{array}{lll}-0.532274 & 2.446147 & -0.000036\end{array}$

$\begin{array}{llll}0.833312 & 2.242441 & -0.000117\end{array}$

$\begin{array}{llll}1.295644 & 0.903167 & -0.000096\end{array}$

$\begin{array}{lll}0.378941 & -0.180443 & 0.000013\end{array}$

$\begin{array}{lll}-1.842694 & -1.054703 & 0.000147\end{array}$

$\begin{array}{llll}2.558066 & 0.409481 & -0.000010\end{array}$

$\begin{array}{llll}1.175711 & -1.305356 & 0.000062\end{array}$

$2.473042-0.927213-0.000482$

$\begin{array}{llll}0.806507 & -2.546355 & -0.000021\end{array}$

$\begin{array}{lll}-3.235422 & -0.834797 & 0.000299\end{array}$

$\begin{array}{lll}-2.521333 & 1.584859 & 0.000126\end{array}$

$\begin{array}{lll}-0.930656 & 3.459751 & 0.000000\end{array}$

$\begin{array}{lll}1.539921 & 3.068961 & -0.000140\end{array}$

$\begin{array}{lll}-3.693377 & -1.827582 & 0.000422\end{array}$

$\begin{array}{lll}-3.561565 & -0.283287 & -0.894817\end{array}$

$\begin{array}{lll}-3.561345 & -0.283162 & 0.895416\end{array}$ 